\title{
KNOWLEDGE MANAGEMENT AS AN ASSISTANT IN DATA PROCESSING AND DATABASE FOR PSYCHOPHYSIOLOGICAL TESTS
}

\author{
Jelena Turlisova \\ Liepaja University, Latvia \\ Anita Jansone \\ Liepaja University, Latvia
}

\begin{abstract}
In nowadays modern society is a knowledge society and we are living in the information communication technologies century, so smart use of different computing tools, instruments and techniques are more and more used and applied for the problems of getting relevant information from the ever-increasing data flow and surrounding information problems resolving. Evaluation of children visual perception is not an exemption. In previous articles, authors described how mechanical skills of children's visual perception can be thoroughly evaluated by computerized system assistance that rates mechanical skills of perception or the computer screening. This computerized approach is a unique and promising opportunity, even a new method, of children's development level of visual perception evaluation at a very early age. In addition - with such evaluation by the computerized system there is not only possible to exclude the risk of the human factor (subjective assess or overlook), but the possibility to get extreme time-saving and extra accuracy of computeraided tests. Extra fast test results proceeding is the main advantage of the computerized psychophysiological tests, especially when computing of tests means technical resolution and value-added outcome of tests results. The main purpose of this study is to consider how the KM system can be used as an assistant in data processing and creation/storage of databases for psychophysiological tests, especially for DTVP tests in the ImageJ computer program.
\end{abstract}

Keywords: database, data mining, data processing, ImageJ knowledge management, Psychophysiological tests.

\section{Introduction}

Knowledge Management (KM) captures, distributes, and effectively uses knowledge. KM systems differ from traditional information systems (IS) by the active role users of KM systems play in building such systems' content. Users of traditional IS are typically not required to contribute to building the content of such systems actively, an effort typically delegated to the managing IS department or to IS consultants. The successful implementation of KM systems requires that their users effectively "use" such systems as traditional IS and 
Turlisova \& Jansone, 2021. Knowledge Management as an Assistant in Data Processing and Database for Psychophysiological Tests

"contribute" to such systems' knowledge base (Becerra-Fernandez \& Sabherwal, 2010). It means that $\mathrm{KM}$ is a process that allows creating, integrating, transforming, applying, and distributing any type and kind of knowledge to satisfy existing or potentially emerging needs and realize new opportunities.

At the same time - different psychophysiological tests mean the concentration of large information volume. This information relates to the data collecting regarding research objects (patients), the theory regarding different test methods and results interpretation, data storage methods, etc. So, as nowadays everything is more and more digitalized, KM system could be used as a digital assistant in data processing and creation and storing of a database for psychophysiological tests, for example - a test that relates to the visual perception, which is a generic term for describing visual information processing techniques such as visual analysis, visual-spatial and visual-motor integration (VMI). Moreover, such psychophysiological tests need a significant database for successful such program working, so there is a need for a particular computer program with KM for successful test processing.

To better understand such a computer program and value-added KM in it, it should be noted that VMI characterizes the level of coordination of visual perception and body movements. I.e., a sensory perception that activates muscles to make any task smooth and productive. Best examples of such activities are catching the ball and writing down from the school board (Schwartz, 2009). Simultaneously, visual perception is a combination of visual, kinaesthetic, and kinetic analyser actions coordinated and simultaneous. It is the ability to perceive, recognize, analyse, systematize, and remember visual information.

Furthermore, if this visual perception process is disturbed, people have many difficulties regarding visual and visual-spatial analysis, for example, difficulty orienting in space or remembering visual images (Tükel, 2013). The most important issue is that children visual perception disorders are not very easily spotted. In most cases only when children experienced problems in learning, such visual perception disorders has been discovered. However, not all people's VMI is fully developed, but they cannot be considered disabled - it is one of the specifics of vision.

As in nowadays modern society is known as a knowledge society and we are living in the information communication technologies century, it is logical that smart use of different computing tools, instruments and techniques are more and more applied for the problems of getting relevant information from the everincreasing data flow and surrounding information problems resolving. Even employers nowadays demand a workforce with a higher level of knowledge and computer literacy (UNESCO, 2005). Moreover, children's visual perception's mechanical skills can also be fully assessed by nowadays solutions - by 
computerized evaluation of mechanical skills of perception or the computer screening. Using a computer program in such an assessment does not eliminate the risk of a human factor. Computer-aided test processing significantly accelerates the collection and acquisition of test results (Howe, Chen, Lee, Chen, \& Wang, 2017). That is why the KM system should be used as an assistant in data processing and creation/storing databases for psychophysiological tests, especially for DTVP tests in the ImageJ computer program.

\section{Tools and Methods}

As the ImageJ computer program was developed to process and obtain the results of the standard psychophysiological test DTVP-2 (Turlisova, 2018), it allows analysing the eye-hand coordination test results of the DTVP-2 test (Exercises 1-16). Scanned filled tests pages or online (with special stylus pen) made test results are proceeding by computer, stored in unique databases that hold quite impressive database/information storage. Furthermore, if those imputed data are smartly used with added KM elements in the program, there are many possibilities to identify particular actions for each test maker to develop his eye-hand coordination and visual perception as a whole in a comparatively short-term period.

Of course, before real test proceedings and results evaluation, much preliminary work should be done - all of those preparation works the author has mentioned in previous researches, such as special macros developing, "etalons" creation and saving for test results evaluation, automatized saving conditions which allow to identify each tested human but not to put all test results in a considerable bind in an anonymous way. It should be noted that extensive and long preparation and the computerized assessment provide a significantly more accurate and faster interpretation of results. Therefore, professionals have more time for the chosen correction works to develop specialized visual perception development materials. (Turlisova \& Jansone, 2019). As the technology world is rapidly developing and updating, the paper aims to consider how KM usage could improve data mining, data processing, and further work with evaluated children's. It is an excellent example for KM and data mining co-working in the real practice to simplify and fasten psychophysiological test results interpretation and decision making to develop visual perception for children whose test results are not acceptable. 
Turlisova \& Jansone, 2021. Knowledge Management as an Assistant in Data Processing and Database for Psychophysiological Tests

\section{Implementation}

ImageJ program works on Android as well as on Windows, based on Microsoft Visual Studio 2017. The purpose is to display on the screen of an electronic tablet (tablet PC) the initial data of psychomotor tests, accept the drawing and its subsequent processing with scoring and saving the results for later comparison. The program can be beneficial for evaluating children, especially children with developmental problems, developing psychomotor skills. So, the program provides different functions, represented in (Fig .1).
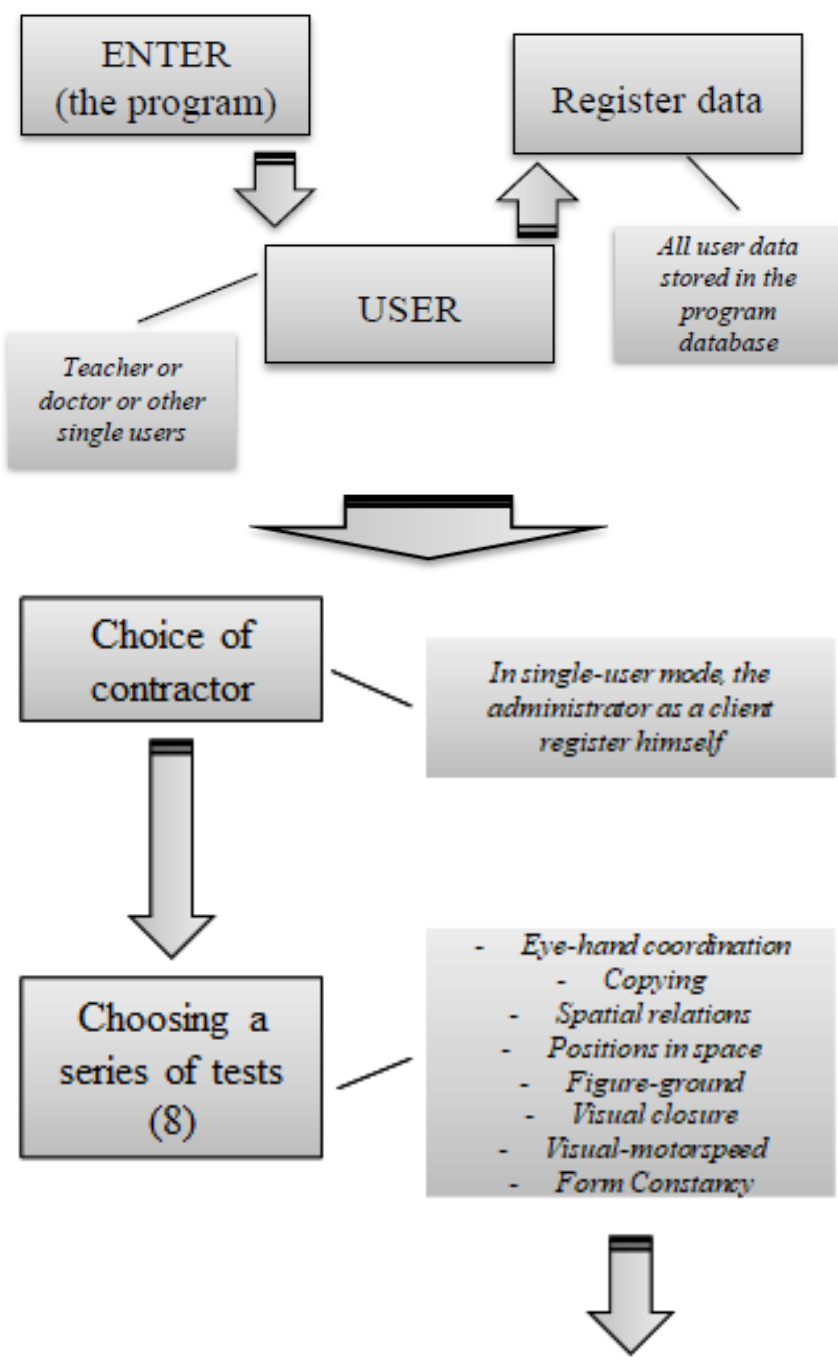

Filling the initial conditions of the next test from selected series to the screen, accounting for the time spent and fixing the end time of the test 


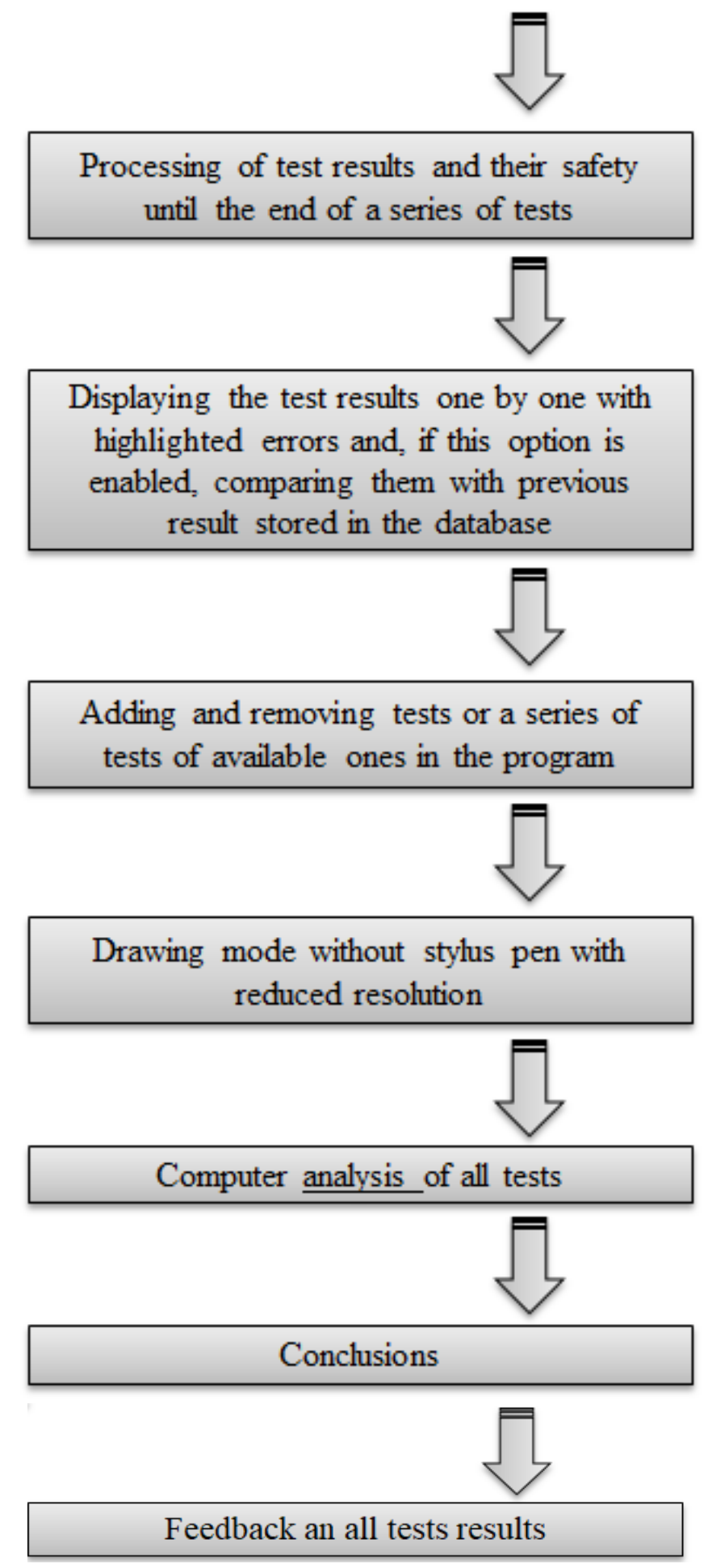

Figure 1 Scheme of ImageJ Program Functions (Turlisova, Jansone, 2019)

For KM's successful implementation in ImageJ and the previously mentioned program prototype working, some data warehouses should be developed. In other words - at the moment, there is an electronic program prototype that allows the analysis of DTVP-2 tests very precisely by unique 
Turlisova \& Jansone, 2021. Knowledge Management as an Assistant in Data Processing and Database for Psychophysiological Tests

algorithms and computer counting methods. Simultaneously, the existing program prototype has some lack of KM in it - without KM existing program only displays results if the tests one by one with highlighted errors and, if this option is enabled, test results could be compared with previous results stored in the database. It is not very usable for fast feedback to decide for further actions from a data mining and KM perspective. Without implementing KM in this program, there are limited possibilities to use all their capacity tests. For example, a test result prescribes specialists' further actions (doctors, teachers, etc.) for possible children's visual perception development. Nevertheless, those action choice options are not collected in one data warehouse where test results can automatically give the tester the most relevant further action scenarios. The authors suggest implementing case-based reasoning (CBR) system/algorithm in the ImageJ program. Initially, some data mining and input for test results should be matched/linked with different further action scenarios related to the different test results. Since there is a great deal of similarity in test results submitted, it is necessary to create a single prototypical case representing the entire set of similar cases, called a protocol. Of course, when there will be base algorithms and scenarios in the program, it will be possible to add new versions of the tests and different action scenarios. In that case, theoretically, the whole world's best practice in the children's visual perception development could be collected in one place.

As KM approach and system as a whole prescribes management of intellectual capital in a computing system, it is important to put in ImageJ program human-based knowledge about visual perception - road-maps, algorithms etc. It will allow using KM as an organised and systemised database for test results proceeding by an integrated approach to information searching, collecting, evaluation, restoring and further distribution. And, even more - if Image J will be used widely by many highly qualified specialists (optometrists) the main database could be updated by knowledge of all of them, not only by one person. All of this could be made by several processes and activities - such as information collecting and codification, human resources connecting with a system (by knowledge), researching by harvesting, harnessing, hunting and hypothesis making. Lessons learned and best practices collecting in one place in Image J as some kind of database is welcomed as well as it is critically important for test-results analysis and right interpretation. It could be possible by using the previously mentioned CBR; - CBR system will compare new test results with similar past tests to determine if any action is needed. The test will be considered as similar if it is for the same child age, similar mistakes, and other -specific aspects. The set of similar test results is then analysed to determine if any further actions for possible children's visual perception development are needed. When discrepancies arise between similar TEST tests, 
the system uses a confidence algorithm to determine what reason it may cause. After the new further action scenario is inputted as an answer to some new test (test result), the database automatically uses it on further tests. A similar approach of CBR system usage is fixed on some insurance companies web pages with the system of- automatic claims to a proceeding, where the system defines what attachments should be added for a different type of claims (Becerra-Fernandez \& Sabherwal, 2010). Of course, after some time of the ImageJ program using in full capacity, such a data warehouse will be huge. It will only mean that DTVP-2 tests made and analysed by computer and artificial intelligence will have a personalized approach for test results, interpreting and finding the best solutions for each child's concrete test results. Although data mining does not require data collection in a data warehouse (BecerraFernandez \& Sabherwal, 2010), the authors think that exactly data mining, that is already inputted in the program, creates data warehouse which could be redesigned to the "Roadmap." As some authors mentioned, 'Roadmap' can mean different things to different people. However, what all those different roadmaps have in common is their goal to help their owners clarify the following three problems: 1) Where are we now? 2) Where do we want to go? 3) How can we get there? (Ma, Liu \& Nakamori, 2006). Moreover, this exactly fits the ImageJ development in line with KM - inputted data. Further data processing with KM assistance can give specialists a clear "Roadmap" with further steps to develop concrete children's visual perception development. For this, according to the author's thoughts, fits two Road mapping Techniques - Expert-based approach and Computer-based approach since those two approaches combines previously defined DTVP-2 test results interpretation methods and KM where high-speed computers, intelligent algorithms, and other modelling tools can assist in estimating and quantifying all test results ever made and further selected options to children's visual perception development.

In summary, the authors can conclude that fully used ImageJ program added with CBR, KM system and the huge (even unique) data warehouse; could open new researches opportunities. DTVP-2 test results interpretation could be correlated to additional information showing new aspects of possibilities/reasons for low developed visual perception.

\section{Conclusions}

There still exists the benefit of having a person in the loop to review and approve DTVP-2 tests, but nowadays, when there are many different computing opportunities - it is very important to use them for very precisely review and approve by unique algorithms and by computing counting methods. After the data mining is complete and the protocols are set up, add KM to the computer 
Turlisova \& Jansone, 2021. Knowledge Management as an Assistant in Data Processing and Database for Psychophysiological Tests

program - and ImageJ will be able to provide static test results and advise further actions. To obtain the highest benefit from data mining in the ImageJ by using $\mathrm{KM}$, there must be a clear statement of the objectives - and it is not only maximally precise test results interpretation, but maximally personalized and admissible suggestions for further actions that are focused on children's visual perception development that could help to develop their skills in different aspects of life, studies, for example, because it is necessary to provide a learning material (chosen by KM from the developed ImageJ system) that is easier to understand or the students/children who have some visual perception problems.

\section{Acknowledgments}

The article was written with the financial support of the European Regional Development Fund project Nr. 1.1.1.5/018 "Pētniecības, inovāciju un starptautiskās sadarbības zinātnē veicināšana Liepājas universitāte.

\section{References}

Becerra-Fernandez, I. \& Sabherwal, R. (2010). Knowledge management: systems and processes. M.E. Sharpe, Inc., 9-10, 113-114.

Howe, T.-H., Chen, H.-L., Lee, C.C., Chen, Y.-D., \& Wang, T.-N. (2017). The Computerized Perceptual Motor Skills Assessment: A new visual perceptual motor skills evaluation tool for children in early elementary grades. Research in Developmental Disabilities, 69, 30-38. doi: 10.1016/j.ridd.2017.07.010

Ma, T., Liu, S. \& Nakamori, Y. (2006). Road mapping as a Way of Knowledge Management for Supporting Scientific Research, Academia Systems Research and Behavioral Science, Syst. Res.23, 743-755. https://doi.org/10.1002/sres.708

Schwartz, S. (2009). Visual Perception: A Clinical Orientation Fourth Edition. McGraw Hill Companies, Pp. 229-241

Tükel, S. (2013), Development of visual-motor coordination in children with neurological dysfunctions. Doctoral thesis. Retrieved from https://openarchive.ki.se/xmlui/handle/ $10616 / 41381$

Turlisova, J. (2018). Eye-hand coordination interaction with visual function. (Acu -rokas koordinācijas saistība ar redzes funkcijām) Masterwork. https://dspace.lu.lv/dspace/ handle/7/39909

Turlisova, J. \& Jansone, A. (2018). E-studies and mastering of educational material for people with visual perception and visual-motor integration problems -topical issues and perspectives. Proceeding of ICLEL, Vol 7. DOI: http://dx.doi.org/10.21533/ pen.v7i1.374

Turlisova, J. \& Jansone, A. (2019). The initial stage of development of a new computer program for the processing of psychophysiological tests. Intelligent Human Systems Integration 2020, 1233-1237. Retrieved from https://link.springer.com/chapter/10.1007/ 978-3-030-39512-4_188

UNESCO. (2005). World report: Towards knowledge societies. Paris: UNESCO Publishing. Retrieved from: http://unesdoc.unesco.org/images/0014/0014001418/141843e.pdf 